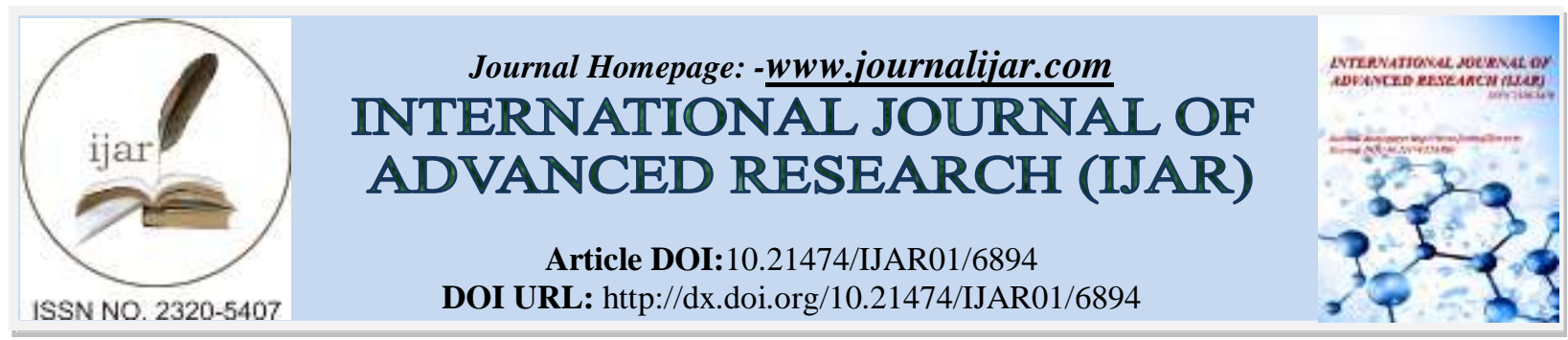

RESEARCH ARTICLE

\title{
HUMAN IMMUNODEFICIENCY VIRUS SEROCONVERSION: RATE AND RETESTING UPTAKE. A CASE OF KAKAMEGA AND NAIROBI COUNTIES, KENYA.
}

\begin{abstract}
Abednego Alibiri Ongeso.
$\mathrm{PhD}$ in Community Health and Development - Reproductive Health (Great Lakes University of Kisumu - Kenya), MSc.N - Maternal and Neonatal Health (Moi, Kenya), BSc.N (Moi, Kenya)Lecturer, School of Nursing Sciences University of Nairobi P.O Box 19676 - 00200 Nairobi.
\end{abstract}

\section{Manuscript Info}

Manuscript History

Received: 09 February 2018

Final Accepted: 11 March 2018

Published: April 2018

Keywords:-

HIV retesting, HIV seroconversion, Elimination of Mother to Child Transmission of HIV, Maternal and neonatal morbidity and mortality, late pregnancy, Serostatus

\section{Abstract}

Background: There is limited empirical data on the prevalence of Human Immunodeficiency Virus (HIV) seroconversion in late pregnancy due to the fact that majority of the women start seeking antenatal care late in pregnancy therefore rarely retest. This leaves a gap in the Elimination of Mother to Child Transmission (eMTCT) strategy thus contributing to high maternal and neonatal morbidity and mortality rates in Kenya.

Objectives: To establish the rate of HIV retesting uptake in late pregnancy and HIV seroconversion rate among women attending Antenatal Clinics in Pumwani Maternity Hospital and Kakamega County Hospital.

Methodology: A longitudinal study design was employed; 1,117 women confirmed as HIV negative in early pregnancy were followed up for three months and retested to ascertain their serostatus. Sample size was obtained through Census and consecutive sampling to recruit individual respondents. Clearance to conduct the study was sought from Ethics Review Committee, Pumwani and Kakamega County Hospitals and the respondents. Researcher administered questionnaires and HIV testing was done for data collection. Descriptive data involved calculation of measures of central tendency. Findings were presented in tables, graphs and narrative.

Results: HIV retesting uptake was 1,073 (96\%) and the rate of HIV serocopositivity among initially HIV negative pregnant women that retested was $8(0.75 \%)$.

Conclusion: HIV retesting uptake is very impressive and the rate of HIV seroconversion is very low. Kenya has made good progress towards eMTCT of HIV.

Copy Right, IJAR, 2018,. All rights reserved.

\section{Study background:-}

\section{Global HIV burden:-}

In 2015, over 36 million people were reported to be living with HIV a virus that causes AIDS worldwide, two-thirds of these people were living in sub-Saharan Africa (Platt et al, 2016). 
Results from the Kenya Aids Indicator Survey (KAIS 2014) indicated that 5.6 percent of Kenyan adults aged 15 to 49 were infected with HIV. The HIV infection rate among women aged 15-49, stood at 6.9 percent, while among men aged 15-49, HIV prevalence stood at 4.2 percent in 2012. This therefore is a clear indicator that women in Kenya bear the greatest burden of HIV infection. This means that the risk of passing on the virus to their unborn babies through Mother to Child Transmission is similarly higher.

\section{HIV testing:-}

HIV testing forms the entry point in the management of HIV and AIDS infection. HIV Testing of women once in ANC seems to be the normal trend in most African countries, this is according to (Derek et al. 2006; Ongeso, 2013; and Gebremeskel et.al 2015); the same fact was also supported by (Rogers et.al 2016).

Kenya has made several strides in so far as HIV testing is concerned, according to (KDHS 2014), 83\% of women in Kenya who tested for HIV received the test results and therefore this was a very good indicator on HIV testing coverage. The Ministry of Health pMTCT Policy Guidelines of 2010 directed that HIV negative women who tested early in pregnancy needed to be retested in the third trimester. This test was supposed to be offered to all women. There is limited empirical data on the number of women who are retested later on in pregnancy according to (Mutiso et.al 2014) who conducted an empirical study among clients drawn from thirty health facilities in Kenya in the year 2013. The study showed that there was very low uptake of repeat HIV retesting in late pregnancy in Kenya. The study further indicated that only $23 \%$ of HIV negative clients returned for the re-test. This trend needs addressing in order to curb MTCT.

\section{HIV seroconversion during pregnancy:-}

HIV seroconversion results mainly from exposure to the virus through sexual intercourse. Risky sexual behavior is the main factor driving HIV infections, according to (Mugo et.al 2011). A study in Nigeria by (Umeononihu et.al 2013) concluded that sexual activity put pregnant women at risk for HIV infection regardless of whether they had single or multiple partners and also regardless of whether they were in polygamous or monogamous unions. Cross generational sex, whereby older men engage with younger women in sex also predisposed these women to HIV infection. These studies bring to the fore the magnitude of missed opportunity associated with a single routine antibody rapid test for pregnant women in early pregnancy. Therefore, routine repeat antenatal HIV testing and counseling in late pregnancy is advocated. Retesting later in pregnancy is important in curbing MTCT according to (Kenyan pMTCT Guidelines 2012).

\section{Statement of the problem:-}

Over the past five years there have been many studies conducted on seropositivity in late pregnancy globally and in Africa and its contribution to the high incidences of Mother to Child Transmission of HIV (Drake et.al, 2014; Steele, 2011; \&McHugh et.al, 2015). In a situation where no child is born with HIV and AIDS, the mortality of children under five years who die of infectious diseases would be greatly reduced from the current rate, which exceeds 3 million deaths per year according to (Requejo \& Bhutta, 2015).

In Kenya however, limited empirical data on seropositivity in late pregnancy exists despite there being a policy directive to retest women in late pregnancy for HIV.

\section{Purpose of the study:-}

The purpose of this study therefore was to find out the uptake of HIV retesting in late pregnancy and the prevalence of HIV seropositivity among pregnant women attending Antenatal Clinics in Nairobi and Kakamega counties.

\section{Justification:-}

The study was conducted in Kakamega and Nairobi counties since they were among the top three leading former provinces in HIV infection rates (KDHS, 2009). The study was therefore instrumental in addressing the problem facing the people of these counties in so far as uptake of repeat HIV testing and counseling is concerned. Pumwani in Nairobi being mainly an urban-slum setting and Kakamega mainly an urban non-slum setting provided a rich source of data in so far as factors affecting eMTCT uptake are concerned. Nairobi and Kakamega counties had almost similar HIV prevalence rates of 11 and 9 percent respectively (KDHS, 2009) and therefore the outcomes from the counties could be compared. Furthermore no similar study has been conducted in the study areas investigating the outcomes of eMTCT in the two counties and highlighting the challenges facing the implementation 
of this program. Furthermore there is limited empirical data on the cohort of women who turn seropositive in late pregnancy in Kenya.

\section{Objectives:-}

1. To determine the proportion of women who undergo retesting among initially HIV negative pregnant women in Kakamega County Hospital and Pumwani Maternity Hospital.

2. To establish the rate of HIV seroconversion among initially HIV negative pregnant women seeking antenatal Care services in Kakamega County Hospital and Pumwani Maternity Hospital.

\section{Literature Review:-}

\section{Proportion of Initially HIV Negative Clients who retest:-}

Retesting Globally:-

Globally, there are limited examples of women retesting perhaps since HIV in developed countries is not a major public health concern.

In a study by (McHugh, et.al 2010), done in the United Kingdom, 2,134 (71\%) of the pregnant women who enrolled in the study underwent retesting in their third trimester. This percentage is low in the context of the need to eliminate HIV considering the fact that majority of the British populace are well informed about the need for HIV testing and retesting.

\section{Retesting in Africa:-}

There are many examples of pregnant women retesting for HIV in late pregnancy in Africa. Generally there is a low uptake of repeat testing of HIV in late pregnancy.

A study by (Heemelaar, Habets, Makukula, Roosmalen \& Akker 2015) in rural parts of Zambia found that there was low uptake of repeat HIV testing with only 79 (24.5\%) of the clients having been tested twice or more during pregnancy. Two issues arose in this study. First the uptake of repeat testing was very low such that it could hardly support the objective of eMTCT. Secondly, just like in Kenya, the uptake of Focused Antenatal Care is low in the rural set up as opposed to the uptake in urban set up and perhaps that is why the uptake of HIV retesting was low in rural Zambia.

Another study indicating low uptake of repeat HIV testing was conducted by (Moodley et.al 2009) and indicated that less than $60 \%$ of respondent took up HIV retesting. Among the 2,377 HIV-negative women retested, $46.2 \%$ and $53.4 \%$ were tested at urban and rural health facilities, respectively. The proportion of clients seeking retesting in this study was very low and as such posed an increased risk of MTCT of HIV during pregnancy and in the postpartum period. One interesting finding about this study is the fact that more rural women were retested as opposed to urban women. The sample size here was large enough and seems representative of the populace. The findings regarding uptake of retesting for HIV was that the levels of uptake was low such that it could not support the objective of virtual elimination of Mother to Child Transmission of HIV.

On the contrary there was improved uptake of HIV retesting in pregnancy as shown in a study done in Nigeria by (Umeononihu et al.,2013), where two hundred and forty five HIV negative attendees were longitudinally recruited into the study. Of these, only 15 (6.12\%) were lost to follow-up giving a retesting rate of 230 (93.88\%). This is extremely high in a longitudinal study and there is a possibility that the researchers may have aggressively followed up the clients to get this high percentage. The high uptake of repeat HIV testing is beneficial in final elimination of MTCT of HIV.

\section{Retesting in Kenya:-}

In Kenya the researcher found that few studies have been done regarding the uptake of repeat HIV testing in late pregnancy. This could be due to the fact that repeat HIV testing in late pregnancy was incorporated in the PNC guidelines less than five years ago and so hospitals are beginning to warm up to the idea. A mixed picture was found regarding uptake of retesting of HIV in late pregnancy among HIV negative women with one study showing a very poor uptake and the other study a very good uptake.

A study by (Kinuthia et.al 2010) in former Western Province and Nairobi revealed that a majority of women in Kenya were willing and ready to be retested. A total of 2,035 (95.3\% of those sampled) women who had tested 
negative before giving birth agreed to be re-tested. This is a very good uptake rate of retesting if the country is to eliminate Mother to Child Transmission of HIV.

A study on the contrary by (Mutiso et.al 2014) revealed very low uptake of repeat HIV retesting in late pregnancy. They collected data from 30 health facilities in Kenya from January to December 2013. Their study indicated that uptake of re-testing in pregnancy remains a significant challenge with only $23 \%$ of HIV-negative clients returning for re-testing $(7,816$ out of 34,103). This poor uptake of HIV retesting in late pregnancy among initially HIV negative pregnant women poses a great challenge and drawback in the fight to virtually eliminate HIV among infants. (Mutiso et.al 2014) study has a good sample size and hence can be generalized well to the Kenyan populace.

\section{Rate of HIV Seroconversion in Pregnancy:-}

Seroconversion is the change of a clients' serum from HIV negative to positive, indicating the development of antibodies in response to infection. Empirical data has revealed that some pregnant women worldwide do seroconvert later on in pregnancy. Studies conducted by (Thaczuk 2009) in Botswana, (Umeononihu et.al 2013) in Nigeria, and (Ongeso 2013) in Kenya have revealed that late pregnancy seroconversions do occur thus bringing to the fore the depravity of missed opportunity associated with a single routine antibody rapid test for pregnant women in early pregnancy. Therefore, routine repeat antenatal HIV testing and counseling in late pregnancy is advocated.

\section{Rate of HIV Seroconversion Globally:-}

The rate of HIV seroconversion globally is higher than reported since the reasons for seroconversion are normally two fold. In one case, the woman may be in window period following infection but pending full seroconversion during her during her initial test. In such a case she may test as HIV negative only to be found to have seroconverted during retest. In the second case she may actually be HIV negative during the first test, after which she may acquire the infection during pregnancy (Ongeso, 2013).

A study by (Drake, Wagner, Richardson, and Stewart 2014) showed that HIV incidence was significantly higher in African than non-African countries (3.6\% versus $0.3 \%$, respectively; $p<0.001$ ) (Drake et al, 2014). The research was a desktop analysis of studies done globally since 1980 to 2013 . Nineteen cohorts with a sample size of 22,803 were used. The study concluded that pregnancy and postnatal periods increase risk of acquisition of HIV.

Another study with almost similar incidence was that by (Paterson et.al 2007) among 443 HIV negative women by antibody result which revealed 15 HIV positive women among them giving an HIV incidence of $3.38 \%$. The study was conducted in Northern Carolina USA revealing that Standard antibody tests do miss acute HIV infection and so algorithms that include pooled HIV RNA testing may improve its detection and represent a further opportunity to prevent MTCT of HIV.

In another study, (Steele 2011), who conducted case studies in the United States of America revealed three cases of HIV positive infants born to mothers who had screened and tested negative for HIV during their first trimesters. The mothers were found to be infected shortly after giving birth upon retest.

However one study differed with all other studies on the issue of seroconversion and this was a study by (McHugh, et.al 2010) done in the United Kingdom, among 2,134 HIV negative pregnant women, where it was discovered that there were no positive third-trimester tests. This is a huge percentage that goes a long way to achieve eMTCT of HIV.

\section{Rate of HIV Seroconversion in Africa:-}

The rate of HIV seroconversion in Africa is high since the biggest burden of HIV is in Sub-Saharan African. Seroconversion in Africa would as well be due to two reasons just like in the case of seroconversion globally. Either, the subjects were in the window period during their first test and tested negative or they acquired the infection during pregnancy.

In Africa, studies have been done to show evidence of women turning HIV positive later on in pregnancy. A study conducted among 400 pregnant women from Tanzania revealed a HIV seroconversion rate of 21 (5.3\%) in late pregnancy (Mbena, Seni, Kajura, Matovelo \& Kihunrwa2014). 
Drake et al (2014), in their systematic review and meta-analysis of seroconversion conducted using 1,176 studies found a HIV incidence in Africa of 3.8\%. The systematic review and met analysis had been conducted in African countries from 1980to 2013. The met analysis showed incidence of infection during pregnancy and postpartum being highest in Southeast Africa (6.2 per 100 person-years), followed by South Africa (4.8 per 100 person-years), East Africa (2.7 per 100 person-years) and West Africa ( 0.7 per 100 person-years). Incidence was significantly higher in southeast Africa compared to West and East Africa.

A study conducted by (Umeononihu et.al 2013), in Nigeria indicated an HIV incidence of 3.9\% among initially HIV negative pregnant women in late pregnancy. This study by (Umeononihu et.al 2013) seemed to give a different HIV incidence than the study by (Drake et.al 2014), and this could be attributed to the study designs employed.

A study by (Moodley et.al 2009) showed a HIV incidence of 3\% among women during pregnancy using a repeat HIV rapid testing strategy during late pregnancy between 36 and 40 weeks of gestation.

A study by Kharsany et.al (2010), who employed HIV-1 RNA pooled nucleic acid amplification testing strategy to screen pregnant women in the "window period" of acute HIV infection in rural South Africa showed an incidence of HIV among 467 HIV negative women to be 4 giving a HIV prevalence rate of $0.9 \%$. A report by (Lu et.al 2009) who conducted a study in Botswana, where repeat HIV testing was offered to 400 women in maternity wards and 244 women seen 9 to 15 months postpartum, all of whom had previously tested negative for HIV during pregnancy revealed an incidence of $1.3 \%$ and $2.9 \%$, respectively.

The authors estimated that $43 \%$ of all infant HIV infections in Botswana in 2008 were attributable to incident maternal HIV infection acquired during pregnancy or postpartum.

From the studies above conducted in Africa among HIV negative women who seroconverted during late pregnancy, showed HIV incidence ranging from $0.9 \%$ to $5.3 \%$ with a mean HIV incidence of $3 \%$. This is a statistic that cannot be wished away since these women may never imagine that they can contribute to eMTCT while in actual sense they will infect not only their children but could also infect their husbands.

\section{Rate of HIV Seroconversion in Kenya:-}

Despite there being limited information on repeat counseling and testing of HIV late in pregnancy in Kenya evidence of women turning positive postpartum has been brought to the fore through a study by (Kinuthia et.al 2010) conducted in the former Nairobi and Nyanza provinces. The study, carried out at six Maternal Child Health clinics in the two former provinces in Kenya, looked at HIV incidence among mothers accompanying their infants for routine immunization. (Kinuthia et.al 2010) reported a 2.6\% HIV incidence among 2,035 women recruited in the study.

In another study by (Ongeso 2013), HIV seroconversion among initially HIV negative pregnant women in Kakamega Provincial General Hospital was 2.98\%. In this study 302 HIV negative pregnant women on initial testing were recruited into the study and retested at least three months after initial test. Among 302 women retested, nine women $(2.98 \%)$ turned HIV positive.

According to (Mutiso et.al 2014), the rate of seroconversion during pregnancy was $1 \%$ (89 out of 7,816). These 89 women stand a high chance of HIV transmission to their infants.

\section{Methodology:-}

\section{Study Design:-}

The researcher employed a descriptive longitudinal study method in data collection. This methodology was considered most appropriate for this study since data was collected over six month period and that the clients needed to be followed up to observe any changes in serostatus three at least three months after initial testing.

The study period was divided into two phases. The first phase of the study was enrollment of HIV negative pregnant clients into the study which took three months. The clients with the help of Research Assistants filled up a questionnaire and those who were not HIV positive were tested for HIV. The information collected included demographic data, respondents' knowledge concerning HIV transmission, among others. 
Phase two involved follow up of the HIV negative clients for retest three months later according to the Ministry of Health eMTCT 2010 guidelines to ascertain whether they had seroconverted or were still seronegative. Those who were seropositive were started on antiretroviral therapy in accordance to the Kenyan Ministry of Health protocols.

\section{Study Site background:-}

All study activities were conducted at Kakamega County Hospital and Pumwani Maternity Hospital, which are the largest government antenatal facilities in Kakamega and Nairobi County. Both hospitals provide antenatal, delivery, postnatal, and family planning services, including antenatal pMTCT and labor ward HIV testing using "opt-out" approach for HIV testing. Repeat HIV testing is also practiced at both facilities.

\section{Study Population:-}

The study population comprised of pregnant HIV negative and HIV positive women attending antenatal clinics at Pumwani Maternity Hospital and Kakamega County Hospital. All HIV negative women in early pregnancy below 26 weeks were enrolled into the study. The HIV negative women were then followed up for three months after which they were retested to ascertain their serology status. All women attending antenatal care below 18 years both married and unmarried were considered as mature minors and included into the study, however consent was sought from their parents before enrolling them into the study.

\section{Exclusion criteria:-}

Pregnant HIV negative women with medical conditions or major complications in pregnancy were excluded from the study.

\section{Sample Size Determination:-}

To account for attrition, the researcher set out to enrol many respondents into the study to carter for the ones who were likely for one reason or another to drop out of the study. Hospital records in June 2013 revealed a total monthly first time attendance of 256 and 213 for Kakamega and Pumwani study sites respectively. In Kakamega site, out of the 256 clients who were first time attendees, only 85 clients turned up for retesting at the facility indicating a $66.8 \%$ drop out rate. In Pumwani site, out of 213 first time attendees, only 71 clients turned up for retesting in the facility also indicating a $66.7 \%$ drop out rate. The researcher therefore decided to use census method so that to enrol clients over a three month period. The researcher decided to use the number of first time attendees to estimate sample size since dropout rate was unpredictable.

The sample size for Kakamega was $256 * 3=768$

The sample size for Pumwani was $213 * 3=639$

Total sample size therefore was $768+639=1,407$ respondents.

\section{Sampling Procedure:-}

Consecutive sampling is a non-probability sampling technique which is considered as the best of all non-probability samples because it includes all subjects that are available that makes the sample a better representation of the entire population(Polit and Beck, 2010).

\section{Pilot Testing:-}

A month prior to commencement of the study, pilot tests were conducted at Mbagathi Sub County Hospital in Nairobi and Bungoma County Hospital during the month of May 2014, to ascertain questionnaire validity and reliability. According to Hertzog (2008) and Connelly (2008) 10\% of the sample size is appropriate for use in a pilot test; the researcher recruited 141 clients for the pilot test. The questionnaire was mainly used to check for the adequacy of the research instruments as follows.

The questionnaire was evaluated for internal consistency whereby Cronbach's alpha of +0.70 was realized and for equivalence the degree of error observed through interrater reliability procedures where the index of equivalence was calculated and found to be strong.

\section{Data Collection Tools:-}

Data collection tools included interviewer administered semi-structured questionnaires. The respondents were given the option of using either English or Kiswahili Questionnaires. HIV Rapid test kits were also used to test the clients to ascertain their serostatus. 


\section{Data collection methods:-}

The respondents were taken through the questionnaire and their responses documented by the research assistant, they were then taken through pretest counseling for HIV before being tested, they were given the results after which post test counseling was done. The HIV test was done to generate data on initial HIV status for all the respondents. A repeat rapid HIV test was done at least three months after the initial test but before delivery to generate data on HIV seroconversion status.

\section{Data Management and Analysis:-}

Participants provided details on demographics, HIV status, sexually transmitted infection (STI) symptoms, sexual activity with their husband and any other sexual partners and their husband's behavior. The quantitative data was captured into Epidata then exported to Statistical Package for Social Sciences (SPSS) Version 20. Measures of central tendency and dispersion were generated for continuous variables for example age and income, while frequency tables displayed categorical variables for example marital status and level of education, among others.

\section{Data Presentation:-}

After analysis of data from the two sites the report generated was in form of tables, graphs pie charts and narration.

\section{Ethical Considerations:-}

The researcher sought ethical approval from Great Lakes University of Kisumu Ethics Review Committee, National Commission for Science and Technology (NACOSTI) and the county executives for Nairobi and Kakamega counties requesting them to allow the researcher carry out the study in their counties. The medical superintendents of the two hospitals were also consulted as well as the ward in charges in the facilities prior to the commencement of the study. Informed consent was sought from adult respondents. For mature minors aged below 18 years, informed consent was sought from the respondents and their parents or guardian as well before engaging them in the study. This was done through detailed informed consent form that was read to the respondents, who in turn signed the consent form, showing that they had understood and agreed to participate in the research.

\section{Limitations:-}

There was scanty empirical literature documented on repeat HIV testing in late pregnancy and as such the researcher ended up using literature for closely related subjects for example, general literature on repeat testing. The number of clients who seroconverted was small that statistical associations could not be done.

\section{Results:-}

\section{Demographic Characteristics}

A total of 1,407 clients were offered a chance to participate in the study; 767 from Kakamega and 640 from Pumwani. Out of this number, 569 and 548 clients accepted to be enrolled into the study thus completed questionnaires and were tested for Kakamega and Pumwani study sites respectively giving an overall response rate of $1,117(79.4 \%)$ i.e. ( $74.2 \%$ vs. $85.6 \%$ for Kakamega and Pumwani sites respectively) as shown in the table 1 below;

Table 1: Acceptance Rate

\begin{tabular}{|l|l|l|l|}
\hline & Kakamega site & Pumwani site & Total \\
\hline Offered the test & 767 & 640 & 1,407 \\
\hline Accepted the test & 569 & 548 & 1,117 \\
\hline Response rate & $74.2 \%$ & $85.6 \%$ & $79.4 \%$ \\
\hline
\end{tabular}

\section{Proportion of Repeat HIV counseling and testing:-}

Respondents upon enrollment were followed up for at least three months after which they were counseled and retested for HIV to establish their serostatus using a rapid HIV test kits. Out of a total of 1,117 HIV negative clients who were followed up for three months a majority 1,073 (96.06\%) turned up for retest. Among the 569 pregnant women who initially tested negative in Kakamega, only 564 (99.12\%) underwent retesting compared to 509 (92.88\%) among 548 in Pumwani study site. Those Lost to follow up therefore were 44 (3.9\%) as shown in the Figure 1 below; 


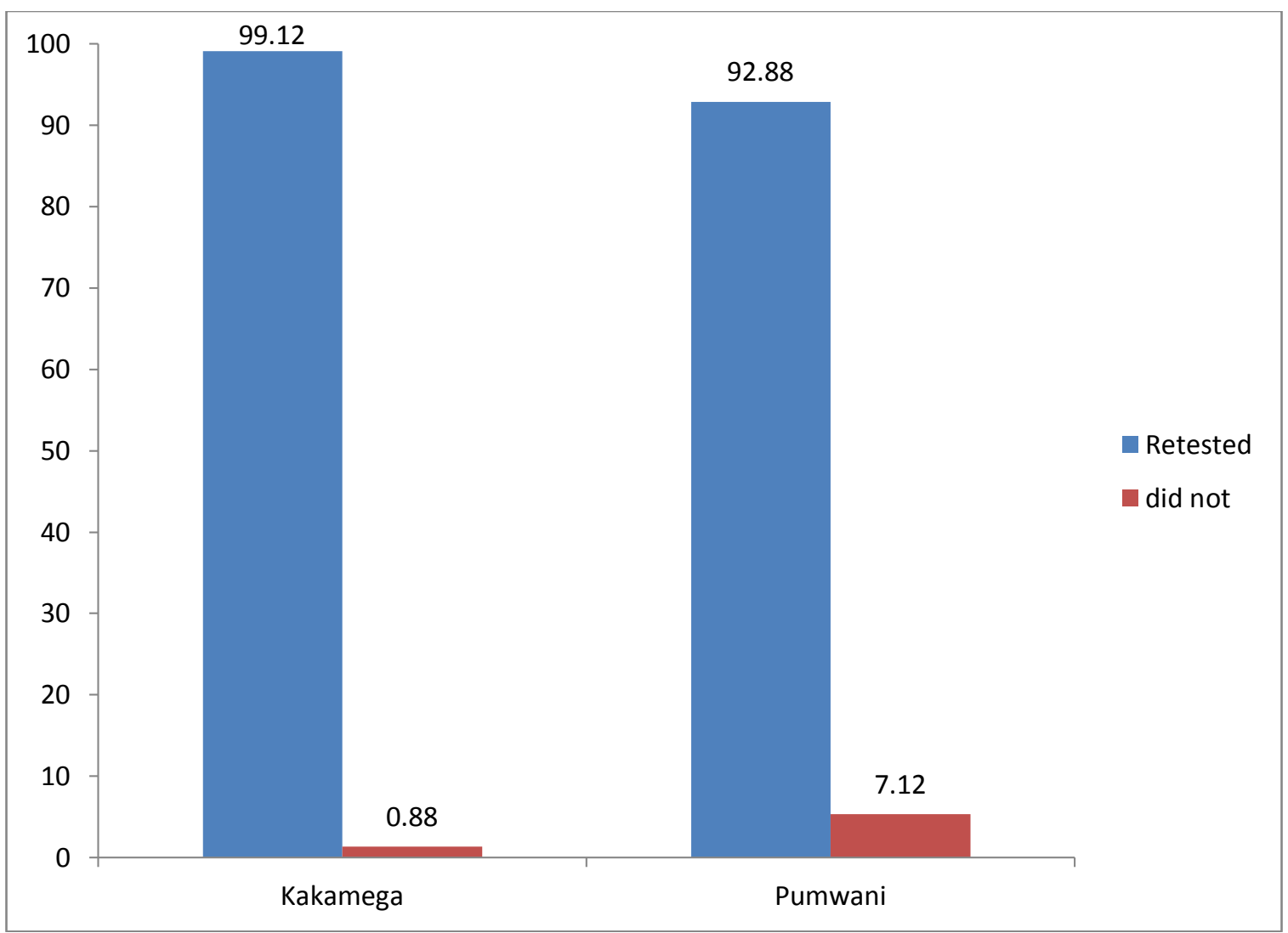

Fig 1:-Re-testing among initially HIV negative pregnant women in Pumwani and Kakamega

Rate of HIV seroconversion in Kakamega and Nairobi:-

Among the 1,073 initially HIV negative pregnant women that retested $8(0.75 \%)$ seroconverted and they were distributed as follows; $3(0.28 \%)$ turned positive on re-test in Kakamega while 5(0.47\%) in Pumwani though the difference in seroconversion rate between the two sites was not statistically significant (Fisher's exact $\chi 2=0.549$, $\mathrm{p}=0.502$ ).

\section{Discussion:-}

\section{Seroconversion among Initially HIV Negative Pregnant Women:-}

Among 1,073 initially HIV negative pregnant women that retested, $8(0.75 \%)$ seroconverted. A majority of them were from Kakamega. This finding is consistent with findings from other studies done by Moschella et.al (2014) in USA, McHugh et.al (2015) in UK, Kharsany et.al (2010) in South Africa and Mutiso et.al (2014) in Kenya whose clients reported HIV incidence of $1 \%$ and below. This is an indicator that indeed the eMTCT interventions are working well in Kenya and that Kenya is well likely to achieve the virtual elimination of MTCT as envisaged by United Nations General Assembly High Level Meeting on AIDS by the year 2015.

In their study, McHugh, et.al (2014) found a HIV seroconversion rate of 0\% among 2,134 clients in the United Kingdom which was a huge leap in not just virtual elimination but rather elimination of MTCT of HIV. This is the Kenyan dream and is possible to be achieved.

These findings contradict those from other studies by Umeononihu et.al (2013) in Nigeria, and Drake et.al (2014) in Africa, Dinh et.al (2015) in South Africa, Moodley, et.al (2009) in South Africa, Paterson, et.al (2007) in USA, Ongeso (2013) in Kenya, Kinuthia et.al (2010) in Kenya and Lu et.al (2009) in Botswana whose findings revealed HIV seroconversion rates of between 1 and $3.9 \%$.

These findings also were divergent to studies by Brubaker et.al (2011) in Kenya and Mbena et.al (2014) in Tanzania whose studies showed HIV seroconversion rates of more than 5\%. These studies are likely to have been done at a time when there was little effort towards eMTCT. 


\section{Conclusion:-}

Uptake of HIV retesting for HIV is very high at more than $96 \%$ and this will lead to early detection of HIV among the population which can lead to quick and efficient management of the pandemic.

Seroconversion was less than $1 \%$ in both sites, which is a good indicator that the country has made big strides towards elimination of mother to child transmission of HIV.

\section{Recommendations:-}

There is need to continuously intensify HIV retesting during late pregnancy in line with Ministry of Health (MOH) guidelines so that to achieve the virtual elimination of Mother to Child Transmission of HIV in line with sustainable development goal 3.

\section{References:-}

1. Brubaker, S. G., Bukusi, E. A., Odoyo, J., Achando, J., Okumu, A., \& Cohen, C. R. (2011). Pregnancy and HIV transmission among HIV-discordant couples in a clinical trial in Kisumu, Kenya. HIV medicine, 12(5), 316321.

2. Moodley, D., Chetty, V., \& Chuturgoon, A. (2012). Evaluation of a 4th generation rapid HIV test for earlier and reliable detection of HIV infection in pregnancy. Journal of Clinical Virology, 54(2), 180-184.

3. Connelly, L. M. (2008). Pilot Studies. Medsurg Nursing, 411-2.

4. Dinh, T. H., Delaney, K. P., Goga, A., Jackson, D., Lombard, C., Woldesenbet, S., \& Shaffer, N. (2015). Impact of maternal HIV seroconversion during pregnancy on early mother to child transmission of HIV (MTCT) measured at 4-8 weeks postpartum in South Africa 2011-2012: a national population-based evaluation. PloS one, 10(5), e0125525.

5. Drake, A. L., Wagner, A., Richardson, B., \& John-Stewart, G. (2014). Incident HIV during pregnancy and postpartum and risk of mother-to-child HIV transmission: a systematic review and meta-analysis. PLoS Med, 11(2), e1001608.

6. Fisher, A. A., Laing, J. E., Stoeckel , J. E., \& Townsend, J. W. (1991). Handbook for Family Planning Operations Research Design 2nd Ed. New York, NY: Population Council.

7. Gebremeskel, F., Dibaba, Y., \& Admassu, B. (2015). Timing of first antenatal care attendance and associated factors among pregnant women in Arba Minch Town and Arba Minch District, Gamo Gofa Zone, south Ethiopia. Journal of environmental and public health, 2015.

8. Guidelines for Prevention of Mother to Child Transmission (eMTCT) of HIV/AIDS in Kenya (3rd Ed) 2009, Available at http://nascop.or.ke/library/eMTCT/eMTCT\%20Guideline-March\%202010.pdf.Accessed 6th Dec 2013

9. Heemelaar, S., Habets, N., Makukula, Z., Roosmalen, J., \& Akker, T. (2015). Repeat HIV testing during pregnancy and delivery: missed opportunities in a rural district hospital in Zambia. Tropical Medicine \& International Health, 20(3), 277-283.

10. Hertzog, M. A. (2008). Considerations in determining sample size for pilot studies. Research in nursing \& health, 31(2), 180-191.

11. KDHS. (2009). Kenya Demographic and Health Survey 2008-2009. Nairobi: Kenya Bureau of Statistics.

12. KDHS. (2014). Kenya Demographic and Health Survey 2014. Nairobi: Kenya National Bureau of Statistics.

13. Kharsany, A. B., Hancock, N., Frohlich, J. A., Humphries, H. R., Abdool Karim, S. S., \& Abdool Karim, Q. (2010). Screening for 'window-period'acute HIV infection among pregnant women in rural South Africa. HIV medicine, 11(10), 661-665.

14. Kinuthia, J., N Kiarie, J., Farquhar, C., Richardson, B., Nduati, R., Mbori-Ngacha, D., \& John-Stewart, G. (2010). Cofactors for HIV-1 incidence during pregnancy and postpartum period. Current HIV research, 8(7), 510-514.

15. Lu, L., Legwaila, K., \& Motswere, C. (2009). HIV incidence in pregnancy and the first post-partum year and implications for PMTCT programs, Francistown, Botswana, 2008. Sixteenth Conference on Retroviruses and Opportunistic Infections. Montreal.

16. Mbena H, Seni J, Kajura A, Matovelo D, Kihunrwa A. Human immunodeficiency virus seroconversion and associated risk factors among pregnant women delivering at Bugando Medical Center in Mwanza, Tanzania. Ann Med Health Sci Res 2014; 4:733-7; doi: 10.4103/2141-9248.141539 Available at: http://wwww.amhsr.org/text.asp?2014/4/5/733/141539 Accessed on 31st January, 2017. 
17. McHugh, E., Costello, M., Phil-Ebosie, A., Le Provost, M., Williams, A., Tilsed, C., \& Brook, G. (2010). Repeat antenatal HIV testing in the third trimester: a feasibility study. HIV Medicine, 11, 66.

18. Ministry of Health (MOH) [Kenya], and ORC Macro (2010).Kenya Demographic and Health survey, Calverton, Maryland: CBS, MOH, and ORC Macro. [Online] Available at: Calverton, Maryland, USA: KNBS and ICF Macro, [online] Available at: http://www.measuredhs.com/pubs/pdf/SR104/SR104KE03.pdf. Accessed 30th March 2012

19. Ministry of Health; Department of Reproductive Health ;( 2012) National Guidelines for Quality Obstetrics and Perinatal Care; Kenya - Government Press

20. Moodley, D., Esterhuizen, T. M., Pather, T., Chetty, V., \& Ngaleka, L. (2009). High HIV incidence during pregnancy: compelling reason for repeat HIV testing. Aids, 23(10), 1255-1259.

21. Moschella, P. C., Hart, K. W., Ruffner, A. H., Lindsell, C. J., Wayne, D. B., Sperling, M. I., ... \& Lyons, M. S. (2014). Prevalence of undiagnosed acute and chronic HIV in a lower-prevalence urban emergency department. American journal of public health, 104(9), 1695-1699.

22. Mugo, N. R., Heffron, R., Donnell, D., Wald, A., Were, E. O., Rees, H., \& Baeten, J. M. (2011). Increased risk of HIV-1 transmission in pregnancy: a prospective study among African HIV-1 serodiscordant couples. AIDS (London, England), 25(15), 1887.

23. Mutiso, P., Towett, R., Aloo, j., Okonji, E., Scheepers, E., Myers, A., Schmitz, K., Simba, M., (2014) Effective monitoring of HIV prevention: retesting HIV-negative pregnant clients 20th International Aids Conference WEPE178 - Poster Exhibition. Available at: http://pag.aids2014.org/abstracts.aspx?aid=7817; Accessed on 29th January, 2016.

24. Ongeso (2013), Incidence of HIV among initially HIV negative pregnant women in Kenya; The Emerging Evidence, Lambert Academic Publishers, Saarbrücken, Germany. Available at: https://portal.dnb.de/opac.htm?method=simpleSearch\&cqlMode=true\&query=idn\%3D1038847737. Accessed on 5th February, 2016.

25. Platt, L., Easterbrook, P., Gower, E., McDonald, B., Sabin, K., McGowan, C., \& Vickerman, P. (2016). Prevalence and burden of HCV co-infection in people living with HIV: a global systematic review and metaanalysis. The Lancet infectious diseases, 16(7), 797-808. Requejo, J. H., \& Bhutta, Z. A. (2015). The post-2015 agenda: staying the course in maternal and child survival. Archives of disease in childhood, 100(Suppl 1), S76S81.

26. Polit, D. F., \& Beck, C. T. (2010). Essentials of Nursing Research: Appraising Evidence for Nursing Practice. New York: Lippincott Williams \& Wilkins.

27. Rogers, A. J., Weke, E., Kwena, Z., Bukusi, E. A., Oyaro, P., Cohen, C. R., \& Turan, J. M. (2016). Implementation of repeat HIV testing during pregnancy in Kenya: a qualitative study. BMC Pregnancy and Childbirth, 16(1), 151.

28. Steele, R. W. (2011). Late pregnancy screening for human immunodeficiency virus. The Pediatric infectious disease journal, 30(1), 72-74.

29. Thaczuk, D., \& Safreed-Harmon, K. (2009, February 13). Breastfeeding in HIV-positive mothers in Botswana did not affect mortality. Retrieved March 3, 2017, from nam-aidsmap: http://www.aidsmap.com/Breastfeedingin-HIV-positive-mothers-in-Botswana-did-not-affect-mortality/page/1433519/

30. Umeononihu, S O., Ikechebelu, J. I., Okonkwo, J. E., Udigwe, G. O., \& Mbachu, I. I. (2013). The prevalence of HIV sero-positivity in late pregnancy among antenatal attendees with seronegative status in first half of pregnancy in Nnewi, South East Nigeria. Journal of HIV and Human Reproduction, 1(1), 25. 